\title{
A New Model for Zero-Order Drug Release I. Hydrophobic Drug Release from Hydrophilic Polymeric Matrices
}

\author{
Ryo Yoshida, Kiyotaka SaKai, Teruo OKano, ${ }^{*, \dagger}$ \\ and Yasuhisa SAKURAI \\ Department of Chemical Engineering, Waseda University, \\ 3-4-1, Ohkubo, Shinjuku-ku, Tokyo 169, Japan \\ *Institute of Biomedical Engineering, Tokyo Women's Medical College, \\ 8-1, Kawada-cho, Shinjuku-ku, Tokyo 162, Japan
}

(Received February 23, 1991)

\begin{abstract}
Conventional monolithic drug delivery devices in which drug is uniformly dispersed typically show square-root-of-time release rates. To achieve zero-order release, monolithic devices of hydrophilic polymer were prepared, loaded with hydrophobic drug. Hydrophobic indomethacin was loaded into hydrophilic matrices of poly( $N$-isopropyl acrylamide(IPAAm)-co-alkyl methacrylate(RMA)) whose hydrophilicity can be varied by temperature without changing the chemical structure. Drug release experiments were performed in phosphate buffered saline. Under conditions of high drug loading in highly hydrophilic polymeric matrices, the release patterns were observed to be nearly zero-order. This result can be explained in terms of increasing diffusivity in polymeric matrices after drug is released, because loaded hydrophobic drug suppresses the swelling of polymeric matrices. A new equation predicting drug release based on diffusivity changes of the polymeric matrices was derived and a good agreement was found between the experimental results and the theoretical release simulation.
\end{abstract}

KEY WORDS Zero-Order Release / Monolithic Device / Poly(2-hydroxyethyl methacrylate) / Poly( $N$-isopropylacrylamide) / Thermoresponsive Polymer / Swelling Kinetics / Case-II Diffusion / Drug Release Kinetics / Drug Release Model /

An important objective of controlled drug release is achievement of maximum efficacy and minimum of side effects of drugs. Often controlled release systems are intended to show "zero-order release", that is, maintaining a constant release rate until exhaustion of drug in the device. Although zero-order release system have not been developed, reservior systems ${ }^{1}$ and osmotic systems ${ }^{2-4}$ have been utilized as partial zero-order release system. In reservoir systems, a core of drug is surrounded by polymeric matrices. Drug release is mediated by diffusion through the surrounding polymeric matrices. Zero-order release is maintained because a constant concentration gradient of drug is kept in the polymeric matrices as long as the reservoir contains a saturated solution and sufficient excess solid drug. In monolithic systems in which drug is uniformly dissolved or dispersed throughout polymeric matrices, however, the drug release rate continuously diminishes with time because of a decrease in concentration gradient at the polymer surface while drug is released. According to diffusion theory, ${ }^{5,6}$ the drug rate is proportional to the square root of time. Consequently, it is difficult to achieve zero-order release with monolithic systems.

\footnotetext{
$\dagger$ To whom correspondence should be addressed.
} 
The design of devices demonstrating zeroorder release rates has been an important subject in pharmaceutical science research. At present, zero-order release is achieved apparently by altering the geometry of the device ${ }^{7,8}$ and by using Case II transport, ${ }^{9-15}$ where drug release is completely governed by the rate of polymer relaxation at the glass/rubbery swelling front. However, a method to achieve zero-order release by regulating drug transport inside polymeric matrices has not yet been established.

Poly( $N$-isopropyl acrylamide) (IPAAm) give a lower critical solution temperature (LCST) in aqueous solution and their networks show high swelling thermosensitivity in water. We have investigated ${ }^{16,17}$ the thermosensitivity of poly(IPAAm) gel by swelling measurement and characterization with a differential scanning calorimeter (DSC). Poly(IPAAm) gel shrinks in water at high temperature and demonstrates a sharp swelling transition in the vicinity of $32^{\circ} \mathrm{C}$. Below this temperature, it swells with decreasing temperature. This means that hydrophilicity of the gel can be controlled by only changing temperature without changing the chemical structure of the polymer. In a monolithic device using the thermosensitive polymer, solute diffusivity in the polymeric matrices can be controlled by changing temperature. We have already achieved complete "on-off" regulation of drug release from IPAAm copolymer gels ${ }^{18-21}$ in response to stepwise temperature change between $20^{\circ} \mathrm{C}$ and $30^{\circ} \mathrm{C}$. It was clarified that the surface skin layer formed with increasing temperature stopped drug release from polymeric matrices.

In this paper, we studied the regulation of drug release rate from IPAAm copolymer gels under constant temperature by the combination of hydrophilic polymer with hydrophobic drug. Hydrophilic polymeric matrices of gel would change to hydrophobic by loaded hydrophobic drug. In this case, drug release from the surface of gel results in the formation of heterogeneous structure between the hydro- philic surface and the hydrophobic inside of gel. So the drug diffusivity in the surface side of gel increases due to high hydrophilicity of polymer in the process of releasing hydrophobic drug. The change of drug diffusivity with time must affect the drug release pattern. Since the hydrophilicity of polymer can be controlled by only changing temperature without changing the chemical structure of the polymer, the degree of diffusivity difference between the surface and inside of gel can be controlled by temperature. If the diffusivity in the surface side of gel became much larger than the inside, overall resistance of drug transport in the gel would decrease with the drug release. This means that the lowering of drug release rate with time is suppressed and zero-order release can be expected. Such an idea would be interesting as the model that leads a new concept to control drug release from polymeric matrics by regulating drug transport in polymeric matrices. The thermosensitive poly(IPAAm) gels as model polymer enable this new concept.

To achieve zero-order release, monolithic devices consisting of hydrophobic drug and hydrophilic polymeric matrices were prepared. A new model of drug release based on the permeability (diffusivity) changes of polymeric matrices versus time was developed. Drug release patterns were simulated on the drug release equations derived from the model. The new concept of controlling drug release rate was discussed from the standpoint of regulating drug transport inside polymeric matrices.

\section{THEORY}

In conventional drug release theory, drug diffusivity in polymeric matrices is assumed to be constant. Nevertheless, the drug loaded in polymeric matrices must act to alter the property of polymer when the property of drug is different from that of polymer. When hydrophobic drug is loaded into hydrophilic matrices with hydrophilic side groups, the 
loaded drug will interact with the hydrophobic backbone chains to decrease the total hydrophilicity of polymeric matrices. Consequently, the drug diffusivity in polymeric matrices decreases because the swelling of polymeric matrices is suppressed. This solute effect would be more remarkable as the amount of loaded drug increases.

In monolithic devices, the amount of drug existing near the surface of the device is relatively small because drug is released gradually from the surface by diffusion. Therefore, the solute effect is small near the surface of device, but remarkable inside the device. This means the drug diffusivity is dependent upon the properties of the polymer near the surface. Drug diffusivity inside the device, however, is mainly regulated by the properties of drug. If both drug and polymer are hydrophilic or hydrophobic, there are no large differences between the diffusivity near the surface and that inside the device. In this case, drug diffusivity in polymeric matrices can be assumed to be constant as in conventional drug release theory. However, if hydrophobic drug is loaded into hydrophilic polymeric matrices, drug diffusivity near the surface becomes larger than that inside the device because of an increasing hydrophilicity in the polymeric matrices after drug is released. In this case, drug diffusivity is not constant in the polymeric matrices, as diffusion from the interior of the polymer matrices becomes rate-limiting.

From this concept of diffusivity change, a new model of drug release has been created. According to the Higuchi model, ${ }^{22}$ two regions exist in the polymeric matrices while drug is released. One is the "dispersion zone" in which solid drug is uniformly dispersed and cannot diffuse. The other is the "dissolved zone" in which drug dissolves in the polymeric matrices and diffuses to the device surface for release. The interface between the dispersion zone and the dissolved zone moves into the matrix interior as drug is released. The dissolved zone (a) t $\leq$ tc

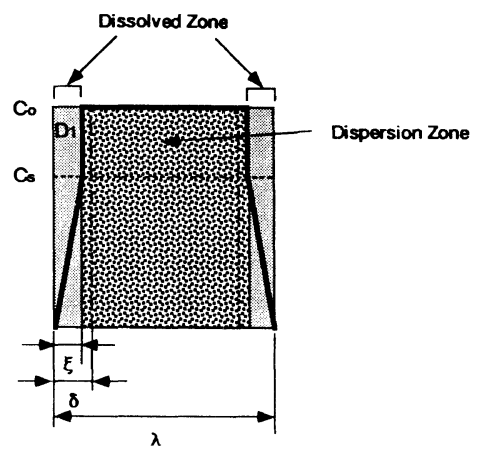

(b) $t>t c$

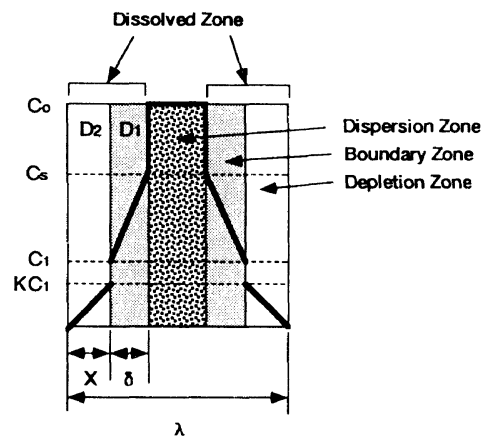

Figure 1. Theoretical concentration profile of drug in polymeric matrices.

has a constant diffusivity in the Higuchi model. But in the model proposed below, the "dissolved zone" consists of "depletion zone" at the surface side and a "boundary zone" between the dispersion zone and the depletion zone. In the depletion zone, very little drug exists in polymeric matrices. Drug diffusivity in this zone becomes larger than that in the boundary zone, because the drug-depleted, hydrophilic polymeric matrices swell and increase drug permeability.

Figure 1 shows the theoretical profile of drug concentration in polymeric matrices with a slab geometry. Drug release from polymeric matrices follows Higuchi model until the thickness of dissolved zone increases to become $\delta$ (Figure 1 (a)). During this period, the release rate is

$$
\frac{\mathrm{d} M_{t}}{\mathrm{~d} t}=\frac{A D_{1} C_{\mathrm{s}}}{\xi}
$$


where $A$ is the surface area of device and $\xi$ is the thickness of dissolved zone. The equation of mass balance at time $t$ is

$$
\frac{2 \xi}{\lambda}=\frac{M_{t}+\left(A \xi C_{\mathrm{s}} / 2\right)}{M_{0}}
$$

where $\lambda$ is the slab thickness. $M_{0}$ is the total amount of drug initially present and can be written as follows.

$$
M_{0}=\frac{A C_{0} \lambda}{2}
$$

Combining eq $1-3$ and integrating give

$$
\begin{gathered}
\xi=\left[\frac{2 D_{1} C_{\mathrm{s}} t}{C_{0}-\left(C_{\mathrm{s}} / 2\right)}\right]^{1 / 2} \\
M_{t}=A\left[D_{1} t C_{\mathrm{s}}\left(2 C_{0}-C_{\mathrm{s}}\right)\right]^{1 / 2}
\end{gathered}
$$

From eq 3 and 5 , the fractional released amount of $\operatorname{drug}(M)$ can be written as follows.

$$
M=\frac{M_{t}}{M_{0}}=2\left[\left(\frac{D_{1}}{D_{2}}\right) T\left(\frac{C_{\mathrm{s}}}{C_{0}}\right)\left(2-\frac{C_{\mathrm{s}}}{C_{0}}\right)\right]^{1 / 2}
$$

where $T$ is dimensionless time which is defined as $T=\left(D_{2} t\right) / \lambda^{2}$. The time when $\xi$ becomes $\delta$, $\left(t_{\mathrm{c}}, T_{\mathrm{c}}\right)$ can be calculated from eq 4 as

$$
\begin{gathered}
t_{\mathrm{c}}=\frac{\delta^{2}}{2 D_{1}}\left[\left(\frac{C_{0}}{C_{\mathrm{S}}}\right)-\frac{1}{2}\right] \\
T_{\mathrm{c}}=\frac{D_{2} t_{\mathrm{c}}}{\lambda^{2}}=\frac{1}{2}\left(\frac{\delta}{\lambda}\right)^{2}\left(\frac{D_{2}}{D_{1}}\right)\left[\left(\frac{C_{0}}{C_{\mathrm{s}}}\right)-\frac{1}{2}\right]
\end{gathered}
$$

From eq 5 and 7 , released amount at $t_{\mathrm{c}}$ is

$$
\begin{aligned}
M_{t_{\mathrm{c}}} & =A\left[D_{1} t_{\mathrm{c}} C_{\mathrm{s}}\left(2 C_{0}-C_{\mathrm{s}}\right)\right]^{1 / 2} \\
& =\frac{A\left(2 C_{0}-C_{\mathrm{s}}\right) \delta}{2}
\end{aligned}
$$

And the fractional released amount at $t_{\mathrm{c}}$ is

$$
M_{\mathrm{c}}=\frac{M_{t_{\mathrm{c}}}}{M_{0}}=\left(\frac{\delta}{\lambda}\right)\left(2-\frac{C_{\mathrm{s}}}{C_{0}}\right)
$$

After the thickness of dissolved zone increases to become $\delta$ and boundary zone is formed $\left(t>t_{\mathrm{c}}\right)$, the dissolved zone are separated to depletion zone (thickness " $x$ ") and boundary zone (constant thickness, " $\delta$ ") (Figure 1 (b)). $D_{1}$ and $D_{2}$ are the drug diffusivities in the boundary zone and the depletion zone, respectively. It can be assumed that $D_{2}$ is greater than $D_{1}$ because of hydrophobic solute effect. The assumptions for pseudo-steady state are as follows: loading concentration $\left(C_{0}\right)$ is greater than drug solubility in the polymeric matrices $\left(C_{\mathrm{s}}\right)$; the concentration profile of drug is linear in the depletion zone and the boundary zone; the boundary zone moves into the interior, keeping a constant thickness, " $\delta$ ". The concept that the boundary between the swollen gel and the glassy core advances interior has already presented as Case-II diffusion for water uptake into polymer gel. ${ }^{23}$ In Case-II diffusion, the swelling polymer gel consists of three zones. Surface which is adjacent to water is a layer of completely swollen gel. Inside this layer there is a fairly thin swelling zone (boundary zone) in which the polymer chains are slowly hydrating and relaxing. Finally there is a layer of unswollen, completely dehydrated rigid polymer matrices. In the process of water uptake, the rate of relaxation of the polymer chains in the swelling zone is the slowest step. The swelling zone moves into the interior at a uniform rate and the water gain increases in direct proportion to time in the case of slab geometry. If the water uptake into polymer exhibits Case-II diffusion, it may be correct for drug release that there are two zones (one is high permeable, another is boundary) in dissolved zone.

From Fick's first law, the release rate is:

$$
\frac{\mathrm{d} M_{\mathrm{t}}}{\mathrm{d} t}=\frac{A D_{2} K C_{1}}{x}=\frac{A D_{1}\left(C_{\mathrm{s}}-C_{1}\right)}{\delta}
$$

where $C_{1}$ is the concentration in the boundary zone at the interface between the depletion zone and the boundary zone. $K$ is partition coefficient between depletion zone and boundary zone. The equation of mass balance at time $t$ is 


$$
\begin{aligned}
M_{t}= & M_{0}-A\left[C_{0}\left\{\frac{\lambda}{2}-(x+\delta)\right\}\right. \\
& \left.+\frac{\delta\left(C_{1}+C_{\mathrm{s}}\right)}{2}+\frac{K C_{1} x}{2}\right]
\end{aligned}
$$

Combining eq 3,11 , and 12 , the following differential equation can be obtained.

$$
\begin{aligned}
M_{t}= & \frac{A D_{1} \delta\left(C_{0}-C_{\mathrm{s}}\right)+A D_{2} K \delta\left(K C_{\mathrm{s}}-C_{0}\right)}{D_{1}} \\
& +\left(\frac{\delta^{2}}{2 D_{1}}\right)\left(1-\frac{K^{2} D_{2}}{D_{1}}\right)\left(\frac{\mathrm{d} M_{t}}{\mathrm{~d} t}\right) \\
& +A^{2} D_{2} K C_{\mathrm{s}}\left(C_{0}-\frac{K C_{\mathrm{s}}}{2}\right) \frac{1}{\left(\mathrm{~d} M_{\mathrm{t}} / \mathrm{d} t\right)}
\end{aligned}
$$

In the case of $D_{1}=D_{2}$ and $K=1$, eq 13 becomes the following equation.

$$
M_{\mathrm{t}}=A^{2} D_{2} C_{\mathrm{s}}\left(C_{0}-\frac{C_{\mathrm{s}}}{2}\right) \frac{1}{\left(\mathrm{~d} M_{t} / \mathrm{d} t\right)}
$$

Integrating eq 14 between $t=0$ and $t=t$ gives

$$
M_{t}=A\left[D_{1} t C_{\mathrm{s}}\left(2 C_{0}-C_{\mathrm{s}}\right)\right]^{1 / 2}
$$

This equation agrees with the Higuchi equation (eq 5) for drug release from a monolithic device when drug diffusivity is constant.

Dividing eq 13 by $M_{0}$ gives the following differential equation.

$$
M=a+b\left(\frac{\mathrm{d} M}{\mathrm{~d} T}\right)+\frac{c}{(\mathrm{~d} M / \mathrm{d} T)}
$$

where $a, b$, and $c$ are constants which are written as follows.

$$
\begin{aligned}
a= & 2\left(\frac{\delta}{\lambda}\right)\left(1-\frac{C_{\mathrm{s}}}{C_{0}}\right) \\
& +2 K\left(\frac{\delta}{\lambda}\right)\left(\frac{D_{2}}{D_{1}}\right)\left(K \frac{C_{\mathrm{s}}}{C_{0}}-1\right) \\
b= & \frac{1}{2}\left(\frac{\delta}{\lambda}\right)^{2}\left(\frac{D_{2}}{D_{1}}\right)\left(1-\frac{K^{2} D_{2}}{D_{1}}\right)
\end{aligned}
$$

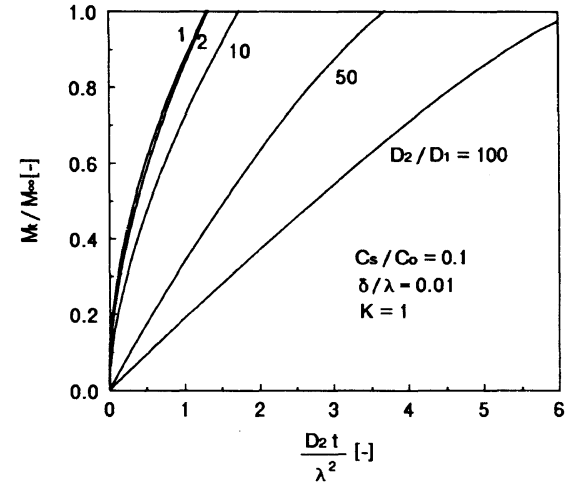

Figure 2. Theoretical fractional release versus dimensionless time for various values of $D_{2} / D_{1}$.

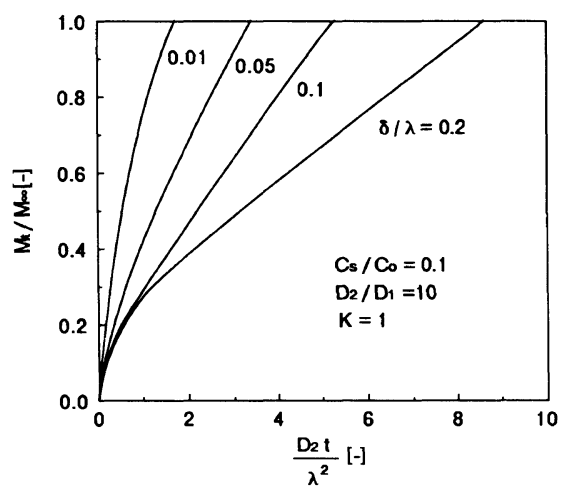

Figure 3. Theoretical fractional release versus dimensionless time for various values of $\delta / \lambda$.

$$
c=2 K\left(\frac{C_{\mathrm{s}}}{C_{0}}\right)\left(2-K \frac{C_{\mathrm{s}}}{C_{0}}\right)
$$

Drug release pattern can be simulated using eq 6 when $T \leq T_{\mathrm{c}}$, and simulated by numerical method using eq 16 and initial condition, eq 8 and 10 when $T \geq T_{\mathrm{c}}$. Figure 2 shows the simulation of drug release patterns for various values of $D_{2} / D_{1}$ in the case that $C_{\mathrm{s}} / C_{0}, \delta / \lambda$ and $K$ are constant. The release pattern for $D_{2} / D_{1}=1$ exhibits square-root-of-time release, but it approaches zero order release as $D_{2} / D_{1}$ increases. Figure 3 shows the release patterns for various values of $\delta / \lambda$ in the case that $C_{\mathrm{s}} / C_{0}$, $D_{2} / D_{1}$ and $K$ are constant. Release pattern approaches square-root-of-time release as $\delta / \lambda$ decreases, which means that the new model 


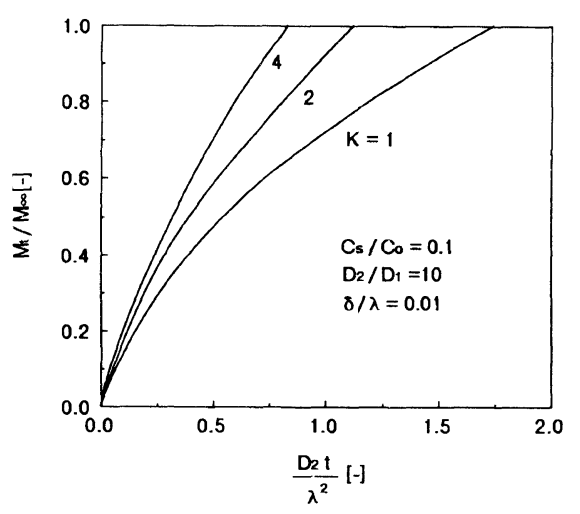

Figure 4. Theoretical fractional release versus dimensionless time for various values of $K$.

agrees with Higuchi model when $\delta / \lambda$ equals to zero. Figure 4 shows the release patterns for various values of $K$ in the case that $C_{\mathrm{s}} / C_{0}$, $D_{2} / D_{1}$ and $\delta / \lambda$ are constant. Release pattern approaches zero-order release as $K$ increases.

\section{EXPERIMENTAL}

Synthesis of Crosslinked Poly(IPAAm-co$R M A)$

Crosslinked random copolymers of $\mathrm{N}$-isopropylacrylamide (IPAAm) (Eastman Kodak Co., Rochester, N.Y.) with alkyl methacrylate (RMA) (3 or $5 \mathrm{wt} \%$ in feed composition) were synthesized using ethyleneglycol dimethacrylate (EGDMA) (Nakarai Chemicals Ltd., Kyoto) as a crosslinker, $t$-butylperoctanoate (BPO) (Nippon Oil and Fats Co., Ltd., Tsukuba, Japan) as an initiator, and distilled 1,4-dioxane (Kanto Chemical Co., Inc., Tokyo) as a diluent. Butyl methacrylate (BMA), hexyl methacrylate (HMA), and lauryl methacrylate (LMA) were used as RMA monomers (Tokyo Kasei Kogyo Co., Ltd., Tokyo). Monomer solutions were bubbled with dried nitrogen for 15 minutes and injected between two Mylar sheets separated by a Teflon gasket $(0.5 \mathrm{~mm})$ and backed by glass plates. The solution was polymerized at $80^{\circ} \mathrm{C}$ for 18 hours. After cooling to room temperature, the membrane was separated from the Mylar sheets and immersed in $100 \%$ methanol for one week to remove all unreacted water insoluble compounds. The methanol was changed every other day. Then the membranes were soaked in $75 / 25,50 / 50$, and $25 / 75 \mathrm{vol} / \mathrm{vol} \%$ methanol/distilled water for one day each. The final washing was pure water for one day.

Swollen membranes were cut into disks ( $15 \mathrm{~mm}$ diameter) using a cork borer and dried ambiently for one day and under vacuum for three days at room temperature.

Synthesis of Crosslinked Poly $(H E M A)$ and
Poly $(H E M A-\operatorname{co}-B M A)$
Crosslinked random copolymers of 2hydroxyethyl methacrylate (HEMA) (Nakarai tesque Inc., Kyoto) with BMA (0 or $5 \mathrm{~mol} \%$ ) were synthesized using EGDMA as a crosslinker and BPO as an initiator without a diluent. The copolymer membranes were polymerized and washed under the same conditions as the case of the poly(IPAAm-coRMA).

\section{Drug Loading}

Dried disks were equilibrated for three days in solutions of indomethacin (Sigma Chemical Co., St. Louis) in ethanol-water $(80: 20$, $\mathrm{vol} / \mathrm{vol} \%$ ). These swollen disks were dried under vacuum for one day at $-20^{\circ} \mathrm{C}$ and for three days at room temperature to prevent drug migration to the surface with rapid evaporation of the solvent. Usually the loaded drug is liable to move to surface side in the process of drying. This migration of drug, however, was prevented by reducing temperature under $-20^{\circ} \mathrm{C}$ in the process of vacuum.

\section{Drug Release}

Indomethacin release experiments were performed in constant-temperature phosphate buffered saline (PBS) (pH 7.4, 11) stirred sufficiently. The device was held in the release media using a wire. Samples of media $(3 \mathrm{ml})$ were withdrawn and replaced with the same amount of PBS at specific time points. The 


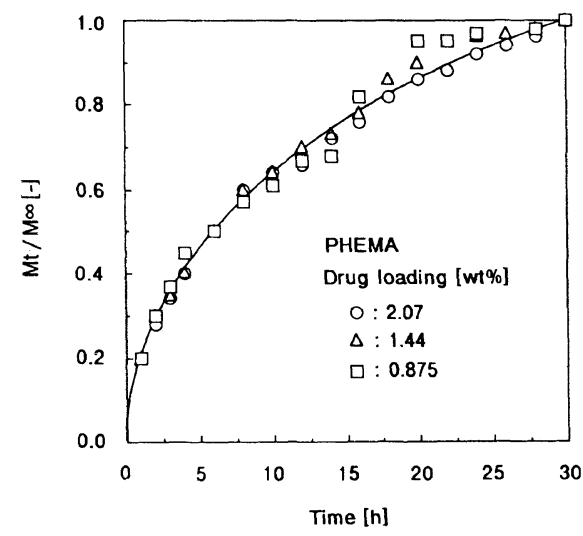

Figure 5. Release profiles of indomethacin from PHEMA at $37^{\circ} \mathrm{C}$.

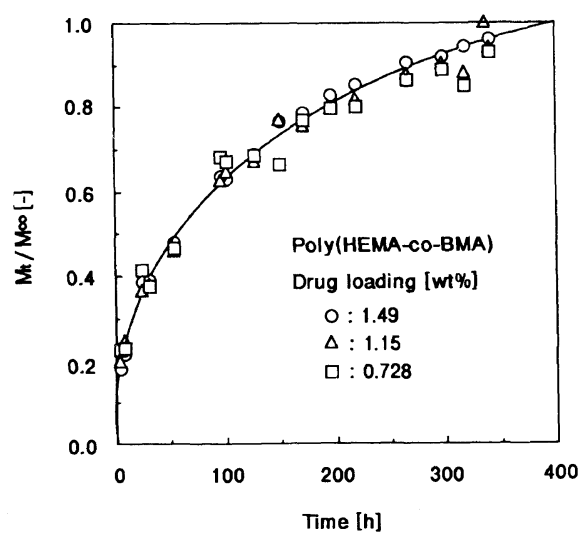

Figure 6. Release profiles of indomethacin from poly(HEMA-co-BMA) at $37^{\circ} \mathrm{C}$.

concentration of indomethacin was measured from the absorbance at $265.9 \mathrm{~nm}$ by UV spectrophotometer (228, Hitachi Ltd., Tokyo).

\section{RESULTS AND DISCUSSION}

Effect of Hydrophilicity/Hydrophobicity of Polymeric Matrices on Drug Release

The equilibrium swelling ratio $\left(W_{\mathrm{H}_{2} \mathrm{O}} / W_{\mathrm{p}}\right)$ in PBS of PHEMA and poly(HEMA-co-BMA) were 0.59 and 0.21 at $37^{\circ} \mathrm{C}$, respectively. Hydrophobicity of the gel was regulated by adding BMA as hydrophobic comonomer to PHEMA. Figures 5 and 6 show the fractional released amount of indomethacin to the total

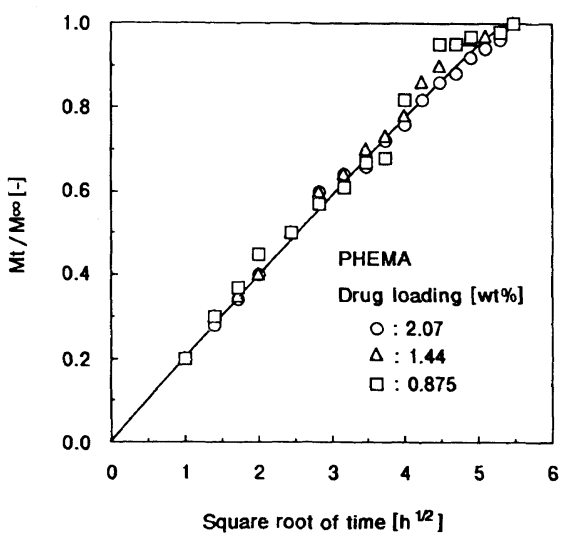

Figure 7. Plot of fractional release versus square root of time for PHEMA.

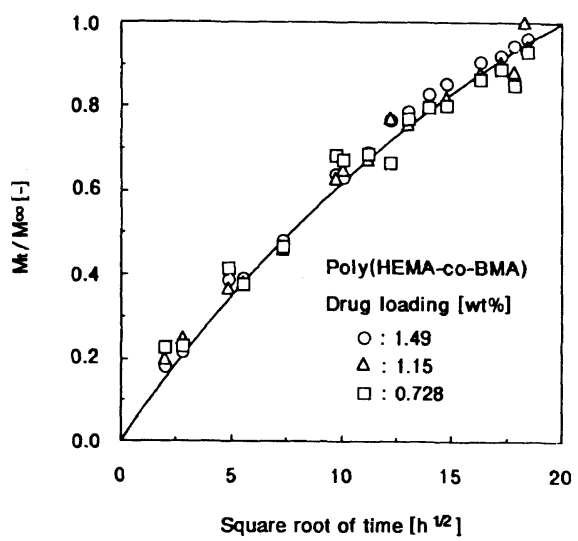

Figure 8. Plot of fractional release versus square root of time for poly(HEMA-co-BMA).

released amount from PHEMA and poly(HEMA-co-BMA) devices at $37^{\circ} \mathrm{C}$. The release rate was observed to diminish continuously with time in both cases. The duration of drug release from poly(HEMA-co-BMA) was longer than that from PHEMA. This result can be explained in terms of decreasing diffusivity with a decrease in water content due to the more hydrophobic monomer (BMA). But the release pattern until exhaustion of drug was the same as that from PHEMA. Figures 7 and 8 show the relationship between the fractional released amount and square root of time for each case. Released drug increased in proportion to square root of time. This relationship between 
the released amount and square root of time was independent of the change in drug loading. These results gave good agreement with the Higuchi theory and suggested that the drug diffusivity was constant in these polymeric matrices. In the case of PHEMA or poly(HEMA-co-BMA), the swelling of polymeric matrices would change little after releasing drug even if the hydrophobic solute effect occur. No large difference exists, therefore, between the diffusivity near the surface (where little drug is present) and that inside the device (where much drug is present). In this case, the drug diffusivity in polymeric matrices can be assumed to be constant as in the conventional drug release theory.

Although poly(IPAAm) gel shows an interesting thermo-responsive properties, it is difficult to apply this gel for drug release device due to poor mechanical properties. So we used copolymer of IPAAm with hydrophobic alkyl methacrylate to increase strength of the gel. Figure 9 shows temperature dependence of the equilibrium swelling ratio for poly(IPAAm-coRMA) (RMA $5 \mathrm{wt} \%$ ) gels. The transition temperature shifted from $32^{\circ} \mathrm{C}$ to $25^{\circ} \mathrm{C}$, but the gel still kept high thermosensitivity in spite of introduction of RMA into poly(IPAAm). Below this transition temperature $\left(25^{\circ} \mathrm{C}\right)$, hydrophilicity of the copolymer gels can be increased by only decreasing temperature without changing the chemical structure of the polymer. These copolymers could be unique model to change hydrophilicity of polymeric matrices by temperature when hydrophobic drug is released. The equilibrium swelling ratio of poly(IAAm-co-BMA) was 1.6 at $20^{\circ} \mathrm{C}$ and indicated that it is more hydrophilic than PHEMA or poly(HEMA-co-BMA).

Figure 10 shows the fractional released amount of indomethacin from more hydrophilic poly(IPAAm-co-RMA) at $20^{\circ} \mathrm{C}$. The release patterns were observed as nearly zeroorder in spite of monolithic device construction. The difference in release patterns between poly(IPAAm-co-RMA) and PHEMA or poly-

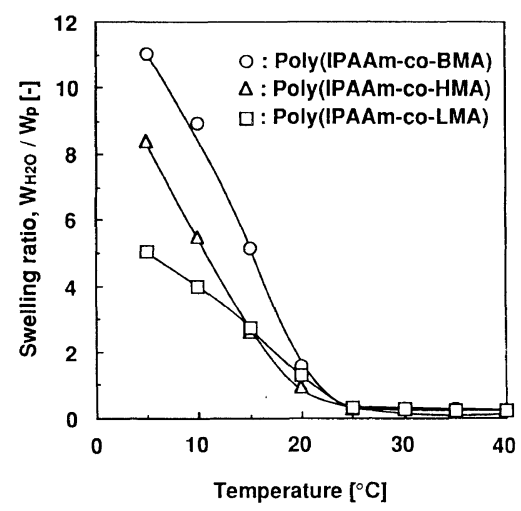

Figure 9. Equilibrium swelling of poly(IPAAm-coRMA) $(95: 5 \mathrm{wt} \%$ ) gels in PBS ( $\mathrm{pH} 7.4)$ as a function of temperature.

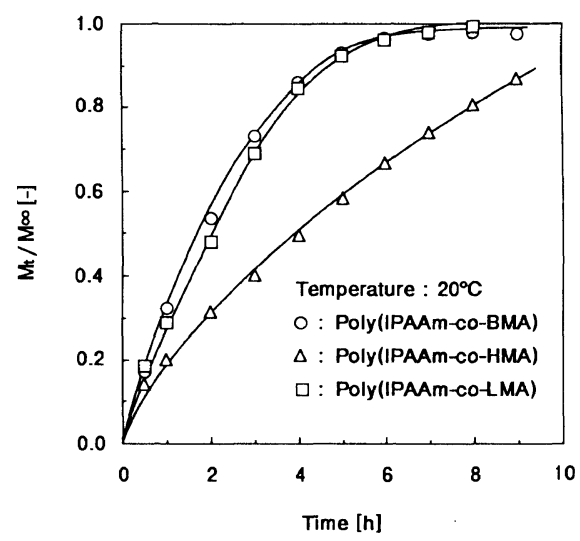

Figure 10. Release profiles of indomethacin from poly(IPAAm-co-RMA) $(95: 5 \mathrm{wt} \%)$ at $20^{\circ} \mathrm{C}$.

(HEMA-co-BMA) can be explained in terms of the diffusivity change due to polymer hydrophilicity as described in the theoretical section. In the case of more hydrophilic poly(IPAAm-co-RMA), polymeric matrices swelled to a greater extent than PHEMA or poly(HEMA-co-BMA) after releasing drug that had suppressed the swelling of polymeric matrices. The drug diffusivity in these swollen matrices (near the surface) became much greater than that inside the device. The decreasing drug concentration gradient at the surface attributed to increasing diffusing distance was compensated by increasing diffu- 


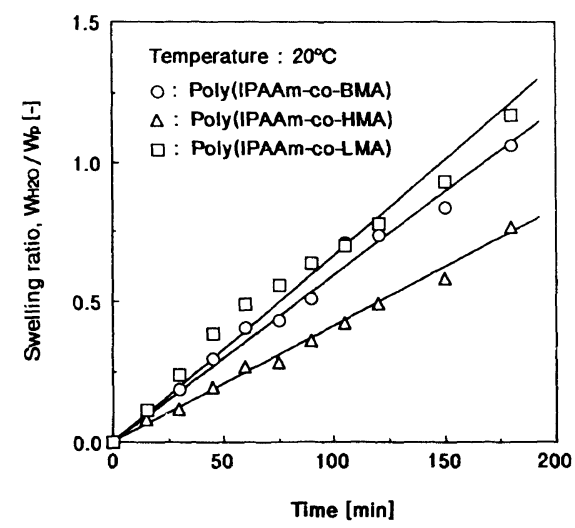

Figure 11. Swelling kinetics of poly(IPAAm-co-RMA) $(95: 5 \mathrm{wt} \%)$ in PBS at $20^{\circ} \mathrm{C}$.

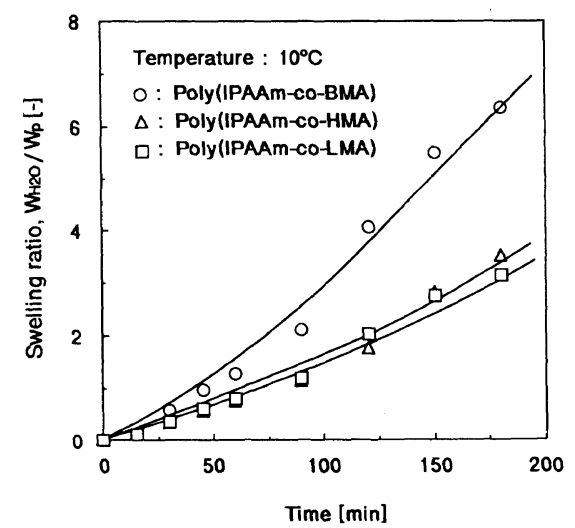

Figure 12. Swelling kinetics of poly(IPAAm-co-RMA) $(95: 5 \mathrm{wt} \%)$ in PBS at $10^{\circ} \mathrm{C}$.

sivity (increased swelling, higher water content). Consequently, the release pattern approached a zero-order rate.

The release rate from poly(IPAAm-coRMA) decreased in the same order as equilibrium $W_{\mathrm{H}_{2} \mathrm{O}} / W_{\mathrm{p}}$ values at $20^{\circ} \mathrm{C}$; poly(IPAAm-co-BMA) > poly(IPAAm-co-LMA) $>$ poly(IPAAm-co-HMA). Poly(IPAAm-coLMA) showed higher swelling than poly(IPAAm-co-HMA) in spite of more hydrophobic (long) alkyl chain. This result suggested that bending configuration due to long alkyl side chains $(\mathrm{RMA}=\mathrm{LMA})$ resulted in more hydration of polymer (that is, higher diffusivity of drug) than expanding configuration due to short alkyl side chains ( $\mathrm{RMA}=\mathrm{HMA}$ ).

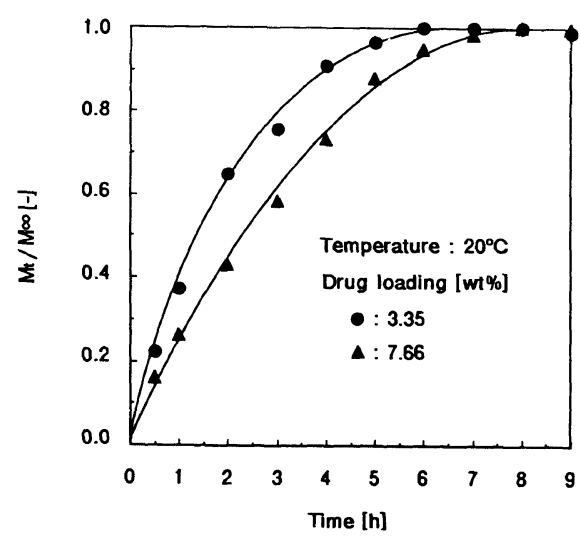

Figure 13. Release profiles of indomethacin from poly(IPAAm-co-BMA) $(97: 3 \mathrm{wt} \%)$ at $20^{\circ} \mathrm{C}$.

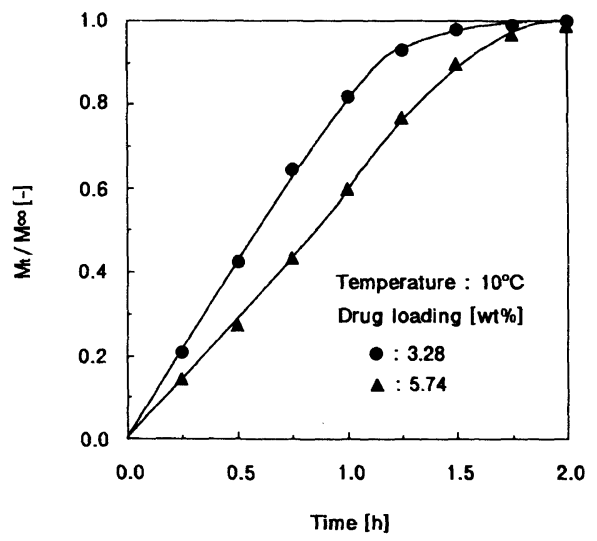

Figure 14. Release profiles of indomethacin from poly(IPAAm-co-BMA) $\left(97: 3 \mathrm{wt} \%\right.$ ) at $10^{\circ} \mathrm{C}$.

\section{Evaluation of the New Drug Release Model}

Figures 11 and 12 show the swelling kinetics of poly(IPAAm-co-RMA) gels at $20^{\circ} \mathrm{C}$ and $10^{\circ} \mathrm{C}$ in PBS. Water gain increased in proportion to time for three gels. This result means that water uptake into the polymer gels obeys Case-II diffusion and there are three zones. (swollen matrix, swelling zone, i.e., boundary zone which moves into interior with keeping constant thickness, and unswollen matrix) in polymer gel. Consequently, it is reasonable to assume the existence of two zones in dissolved zone for drug release as the new model described in theory section.

Figures 13 and 14 show the fractional 
released amount of indomethacin from poly(IPAAm-co-BMA) at $20^{\circ} \mathrm{C}$ and $10^{\circ} \mathrm{C}$. When the temperature is constant at $20^{\circ} \mathrm{C}$ or $10^{\circ} \mathrm{C}$, the release pattern approached zero-order release as the drug loading increased. When the drug loading is constant, the release pattern approached zero-order release as the temperature decreased from $20^{\circ} \mathrm{C}$ to $10^{\circ} \mathrm{C}$. These results can be explained in terms of the new release model derived herein. According to this model, the release pattern approaches zeroorder release as the value of $D_{2} / D_{1}$ increases as shown in Figure 2. The diffusivity of drug in the boundary zone $\left(D_{1}\right)$ decreases with an increase in drug loading due to the solute effect of the hydrophobic drug loaded in this zone. The diffusivity in the depletion zone $\left(D_{2}\right)$ is kept constant when temperature is constant, because the swelling of polymeric matrices in this zone does not change. Therefore, the value of $D_{2} / D_{1}$ increases with an increases in drug loading at constant temperature. Consequently, the release pattern approaches zeroorder release with an increase in drug loading at constant temperature as shown in Figures 13 and 14.

The equilibrium swelling of the polymer increases with a decrease in temperature. The swelling ratio $\left(W_{\mathrm{H}_{2} \mathrm{O}} / W_{\mathrm{p}}\right)$ of poly(IPAAm-coBMA) at $10^{\circ} \mathrm{C}$ is about five times as large as that at $20^{\circ} \mathrm{C}$. In the new model, $D_{2}$ increases with an decrease in temperature due to the swelling in the depletion zone. $D_{1}$ is kept constant when drug loading is constant because the swelling of polymeric matrices does not change in the boundary zone. Therefore, the value of $D_{2} / D_{1}$ increases with a decrease in temperature at constant drug loading. Consequently, the model predicts the release pattern to approach a zero-order rate with an decrease in temperature at constant drug loading as shown in Figures 13 and 14.

These experimental results demonstrate the validity of the new model on the basis of diffusivity changes in polymeric matrices. The release pattern can be regulated by changing the swelling of polymeric matrices and drug loading. To achieve zero-order release, the value of $D_{2} / D_{1}$ must be made large by using high swelling polymeric matrices and loading a large amount of hydrophobic drug into the matrices. Such a method to achieve zero-order release by regulating the drug transport inside polymeric matrices has not been reported in conventional controlled release technology. The theory created here fundamentally supports the new concept of controlling drug release rates from monolithic devices.

\section{CONCLUSIONS}

(1) Thermosensitive poly(IPAAm-coRMA) copolymer gels were capable of the drug release model to change swelling (hydrophilicity) of polymeric matrices by temperature without changing the chemical structure.

(2) A new model of drug release was developed on the basis of diffusivity changes in polymeric monolithic devices consisting of hydrophobic drug and hydrophilic polymeric matrices.

(3) The theoretical release patterns were simulated based on our model. The validity and predictive capabilities of the model were demonstrated by the experimental results in various copolymer systems.

(4) The drug diffusivity in the surface side of gel increases due to high hydrophilicity of polymer in the process of releasing hydrophobic drug. Therefore, overall resistance of drug transport in the gel decreases with drug release and the release pattern approaches zero-order release.

(5) A new concept for controlling drug release rate from monolithic devices by regulating drug transport in polymeric matrices was obtained from this model.

Acknowledgement. The authors gratefully acknowledge Dr. David W. Grainger for valuable discussions. This research was supported partially by a Grant-in-Aid for 
Scientific Research from the Ministry of Education, Science, and Culture of Japan (New Functionality Materials-Design, Preparation, and Control), and Japan Research Promotion Society for Cardiovascular Disease.

\section{NOMENCLATURE}

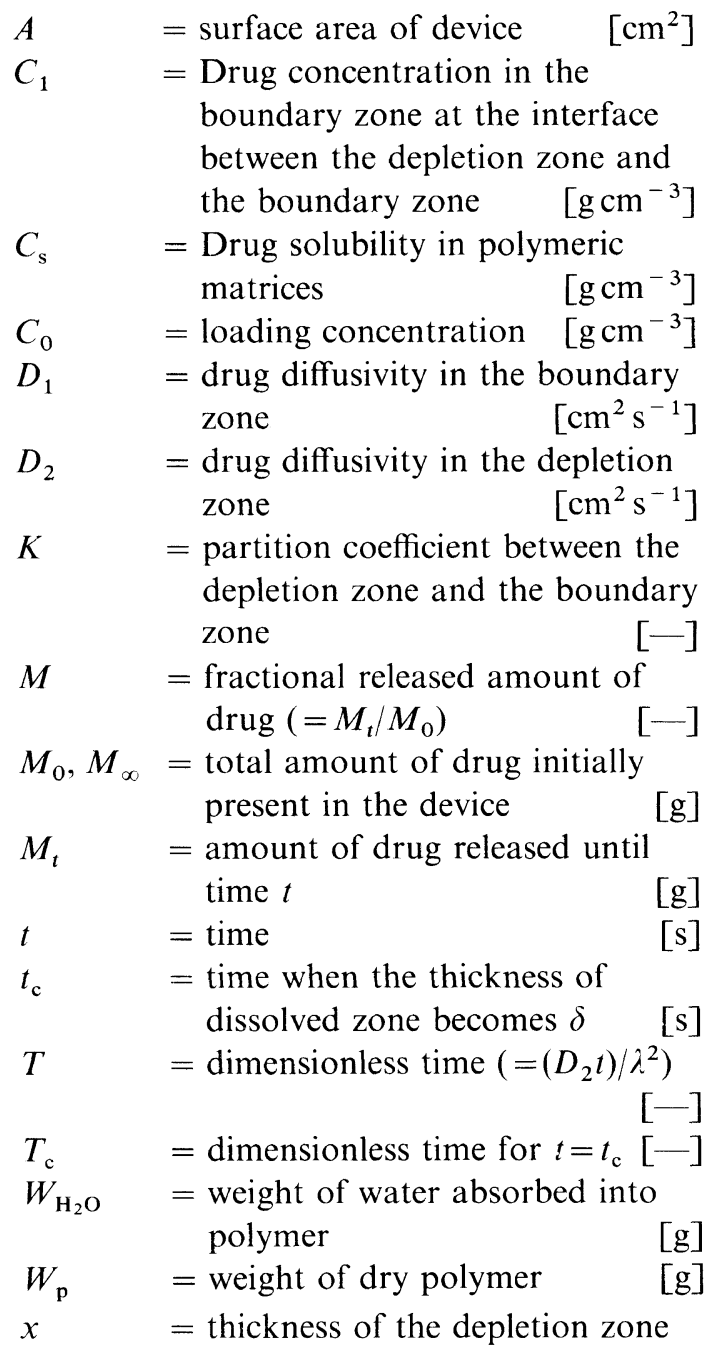

$[\mathrm{cm}]$

Greek Symbol

$\begin{array}{llr}\delta & =\text { thickness of the boundary zone } \\ \lambda & =\text { slab thickness } & {[\mathrm{cm}]} \\ \xi & =\text { thickness of dissolved zone when }\end{array}$

$$
t \leq t_{\mathrm{c}}
$$

$[\mathrm{cm}]$

\section{REFERENCES}

1. T. Okano, M. Miyajima, F. Komada, S. Imanidis, S. Nishiyama, S. W. Kim, and W. I. Higuchi, J. Control. Rel., 6, 99 (1987).

2. F. Theeuwes, J. Pharm. Sci., 64, 1987 (1975).

3. F. Theeuwes and S. I. Yum, Ann. Biomed. Eng., 4, 343 (1976).

4. F. Theeuwes, "Delivery of Active Agents by Osmosis," In Controlled Release Technologies: Methods, Theory and Applications, A. G. Kydonieus, Ed., CRC Press, Boca Raton, FL, 1980, p 195.

5. J. Crank, "Mathematics of Diffusion," Oxford University Press, London, 1975, p 47.

6. R. W. Baker, "Controlled Release of Biologically Active Agents," Wiley-Intersceince, New York, N.Y., 1987, p 39.

7. D. Brooke and R. J. Washkuhn, J. Pharm. Sci., 66, 159 (1977).

8. R. A. Lipper and W. I. Higuchi, J. Pharm. Sci., 66, 163 (1977).

9. H. B. Hopfenberg and K. C. Hsu, Polym. Eng. Sci., 18, 1186 (1978).

10. H. B. Hopfenberg, A. Apicella, and D. E. Saleeby, J. Membrane Sci., 8, 273 (1981).

11. R. W. Korsmeyer and N. A. Peppas, J. Membrane Sci., 9, 211 (1981).

12. R. W. Korsmeyer and N. A. Peppas, "Macromolecular and Modeling Aspects of Swellingcontrolled Systems," In Controlled Release Delivery Systems, T. J. Roseman and S. Z. Mansdorf, Ed., Marcel Dekker, Inc., New York, N.Y., 1983, p 77.

13. R. W. Korsmeyer and N. A. Peppas, J. Control. Rel., 1, 89 (1984).

14. P. I. Lee, Polym. Commun., 24, 45 (1983).

15. P. I. Lee, J. Control. Rel., 2, 227 (1985).

16. Y. H. Bae, T. Okano and S. W. Kim, J. Polym. Sci., B, Polym. Phys., 28, 923 (1990).

17. Y. H. Bae, T. Okano, and S. W. Kim, Pharm. Res., 8, 531 (1991).

18. T. Okano, Y. H. Bae, H. Jacobs, and S. W. Kim, $J$. Control. Rel., 11, 255 (1990).

19. Y. H. Bae, T. Okano, and S. W. Kim, Makromol. Chem., Rapid Commun., 8, 481 (1987).

20. Y. H. Bae, T. Okano, and S. W. Kim, Pharm. Res., 8, 624 (1991).

21. T. Okano, Y. H. Bae, and S. W. Kim, "TemperatureResponsive Controlled Drug Delivery," In Modulated Control Release System, J. Kost, Ed., CRC Press, Boca Raton, FL, 1990, p 17.

22. T. Higuchi, J. Pharm. Sci., 50, 874 (1961).

23. T. Alfrey, Jr., E. F. Gurnee, and W. G. Lloyd, $J$. Polym. Sci., C, 12, 249 (1966). 